\title{
INVERSIÓN EN INFRAESTRUCTURA Y CRECIMIENTO ECONÓMICO, RELEVANCIA DE FACTORES INSTITUCIONALES ${ }^{1}$
}

\author{
INFRASTRUCTURE INVESTMENT AND ECONOMIC GROWTH, RELEVANCE OF \\ INSTITUTIONAL FACTORS
}

INVESTIMENTO EM INFRAESTRUTURA E CRESCIMENTO ECONÔMICO, RELEVÂNCIA DE FATORES INSTITUCIONAIS

\section{Manfred Esquivel Monge ${ }^{2}$ \\ Kerry Loaiza Marín ${ }^{3}$}

\begin{abstract}
Resumen
Se cuantifica el efecto que tiene la inversión en infraestructura sobre el crecimiento económico y si está condicionado por factores de índole institucional mediante modelos dinámicos de panel con variables instrumentales y técnica GMM. Los resultados son coherentes con estudios previos en cuanto a la magnitud del efecto de la infraestructura sobre el crecimiento. Se halla evidencia de que factores institucionales inciden significativamente en el potencial efecto expansivo de la infraestructura. Se encuentra que países de América Latina estarían limitando el potencial expansivo de su inversión en infraestructura al presentar indicadores de desempeño institucional desfavorables. Lo mismo estaría sucediendo con países de ingreso medio y bajo.
\end{abstract}

Palabras clave: infraestructura; crecimiento; instituciones; libertad económica; corrupción.

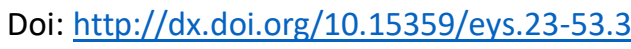

Fecha de recepción: 04-12-2017. Fechas de reenvíos: 13-03-2018, 02-04-2018. Aceptado el 04-04-2018. Publicado el 06-04-2018.

${ }^{1}$ Las ideas expresadas en este documento son de los autores y no necesariamente representan las del BCCR.

${ }^{2}$ Máster en Economía, Essex University, Reino Unido. Investigador del Departamento de Investigación Económica del Banco Central de Costa Rica (BCCR), Costa Rica. Correo electrónico: esquivelmm@bccr.fi.cr

${ }^{3}$ Candidato a Doctor, Université Toulouse, Francia. Investigador del Departamento de Investigación Económica del Banco Central de Costa Rica (BCCR), Costa Rica. Correo electrónico: loaizamk@bccr.fi.cr 40

Manfred Esquivel Monge y Kerry Loaiza Marín

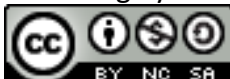

Revista Economía y Sociedad by Universidad Nacional is licensed under a CreativeCommons Reconocimiento-NoComercial- 


\begin{abstract}
We quantify the effect of infrastructure investment on economic growth and test whether that effect is conditioned by institutional factors. This is done by estimating dynamic panel data models with instrumental variables using GMM estimation. Results are in line with those reported by previous studies in terms of the magnitude of the effect of infrastructure on economic growth. Evidence suggests that bad institutions lessen the potential expansive effect of infrastructure investment. Latin American countries would be restraining the expansive potential of their infrastructure investment by showing unfavorable institutional performance. The same would be happening with mid and low-income countries.
\end{abstract}

Key words: Infrastructure; growth; institutions; economic freedom; corruption.

\title{
Resumo
}

Quantifica-se o efeito que o investimento tem em infraestrutura sobre o crescimento econômico, e se está condicionado por fatores de índole institucional através de modelos de painéis dinâmicos com variáveis instrumentais e técnica GMM. Os resultados são coerentes com estudos prévios quanto à magnitude do efeito da infraestrutura sobre o crescimento. Há evidências de que os fatores institucionais têm impacto significativo no potencial efeito expansivo da infraestrutura. Verifica-se que países da América Latina estariam limitando o potencial expansivo de seu investimento em infraestrutura, apresentando indicadores de desempenho institucional desfavoráveis. O mesmo estaria acontecendo com países de baixa e média renda.

Palavras-chave: Infraestrutura; crescimento; instituições; liberdade econômica; corrupção.

\section{Introducción}

El tema del impacto de la inversión en infraestructura sobre el crecimiento económico es de gran relevancia en un contexto en el que la recuperación económica mundial aún no se considera definitiva; las bajas tasas de interés nominales imprimen un panorama de facilidad para el financiamiento de inversión productiva en infraestructura y las cifras de desempleo se resisten a mejorar. En Costa Rica gran parte del rezago en infraestructura que se ha documentado ampliamente (Castro y Porras, 2009; Arce, Céspedes y Jiménez, 2006; Mesalles, 2010; Mesalles y Celis, 2011) se atribuye a razones de índole institucional. Si bien la literatura empírica respalda la 
existencia de una relación positiva entre la infraestructura y el crecimiento económico (Aschauer, 1989; Canning, 1999; Calderón y Servén, 2004), resulta importante valorar si ese impacto está condicionado por la calidad institucional de los países. El objetivo de esta investigación es cuantificar el efecto que tiene la infraestructura sobre el crecimiento económico y evaluar si tal efecto está condicionado por factores institucionales.

La disminución a nivel mundial del protagonismo del sector público como proveedor de infraestructura, acaecida con mayor vigor en la década de los ochenta, generó una primera generación de literatura sobre el efecto de la infraestructura en el crecimiento. Aschauer (1989) es quizá el más citado y concluye, en primer término, que el acervo de capital público no militar es más importante en la determinación de la productividad que el flujo de gasto no militar o el gasto militar. Además, comprueba que el acervo de estructuras de capital (carreteras, alcantarillado y sistemas de provisión de agua potable) tiene mayor poder explicativo de la productividad que el acervo de otros equipos.

Ferreira (1995) provee un modelo teórico microfundado que respalda la participación del gobierno en la provisión de infraestructura como complemento a la efectuada por un sector privado. Tal provisión incrementa el retorno de las inversiones del sector privado y conduce a una expansión de la actividad empresarial doméstica por parte del sector privado más pobre. El modelo justifica la inversión del gobierno en sectores con elevadas externalidades positivas, pues puede mejorar tanto la equidad como la eficiencia.

Calderón y Servén (2002 y 2004) y Calderón, Easterly y Servén (2003) son algunos de los trabajos más relevantes que enfatizan el caso de los países de América Latina. En el documento del 2002 se concluye que la brecha en infraestructura explica una considerable fracción de la brecha en producción de los países de América Latina respecto a economías emergentes del este asiático en los años ochenta y noventa. En el estudio de 2004 se analiza el efecto de la infraestructura sobre la distribución de la riqueza, encontrando que la desigualdad disminuye tanto con cantidad como con la mejor calidad de la infraestructura. En ambos estudios se estiman modelos dinámicos de datos panel tratando problemas econométricos con variables instrumentales y el método generalizado de momentos (GMM), en línea con Arellano y Bond (1991), Arellano y Bover (1995) y Blundell y Bond (1998).

Auerbach y Gorodnichencko (2011 y 2012) estudian el tamaño de los multiplicadores fiscales en economías en recesión mediante modelos de cambio de régimen. Documentan multiplicadores mayores en las recesiones que en las expansiones. Basándose en esta metodología, el Fondo Monetario Internacional (FMI, 2014) presenta un análisis sobre los efectos macroeconómicos de la inversión pública. Se concluye que aumentos en la inversión pública en infraestructura incrementan la producción tanto a corto como a largo plazo, especialmente si la economía muestra capacidad ociosa. Se indica, además, que, de cumplirse esta última condición, y si se 42

Manfred Esquivel Monge y Kerry Loaiza Marín

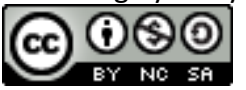

Revista Economía y Sociedad by Universidad Nacional is licensed under a CreativeCommons Reconocimiento-NoComercialCompartirlgual 4.0 Internacional License.

Creado a partir de la obra en http://www.revistas.una.ac.cr/index.php/economia 
identifican con claridad las necesidades de infraestructura, los proyectos financiados con deuda podrían tener efectos positivos significativos sobre el crecimiento sin afectar la relación deuda/PIB.

Celis (2007) cuantifica el impacto de la inversión pública en infraestructura en Costa Rica con base en información georreferenciada de la Encuesta Nacional de Ingresos y Gastos, la aplicación de análisis de regresión y propensity score matching. Reporta que el mayor y mejor acceso a los mercados de agua, electricidad y teléfono aumenta el número de horas trabajadas por hogar, estimula la reasignación de la fuerza de trabajo desde actividades agrícolas a actividades no agrícolas e incrementa el ingreso de los hogares.

Varios estudios documentan el estancamiento de la inversión en infraestructura, especialmente de carácter público, acaecida en los últimos 30 años en Costa Rica. Arce, Céspedes y Jiménez (2006) señalan que sin bien la desaceleración del crecimiento económico que se dio en los noventa y primera mitad de los dos mil se debe mayoritariamente a factores externos, el deterioro de la inversión en infraestructura puede considerarse entre los principales causantes internos. Además, señalan que esa desaceleración coincide con el estancamiento en la reducción de la pobreza en el país.

Castro y Porras (2009) argumentan que tal deterioro en la calidad y cobertura de la infraestructura ocurrida a partir los ochenta, obedece al estancamiento de la inversión pública que resultó de las fuertes restricciones presupuestarias heredadas de las crisis de deuda de esa década. Mesalles (2010) señala que entre los años 2000 y 2007 la inversión pública en infraestructura para transportes fue inferior al $1 \%$ del PIB. Para el mismo periodo, países con similar nivel de desarrollo invirtieron en promedio más del $2 \%$ del PIB.

Podría argumentarse que Calderón y Servén (2002 y 2004) proveen respaldo empírico a las argumentaciones de Arce, Céspedes y Jiménez (2006); sin embargo, existe un tema que aquellos autores no tratan y que puede constituir una valiosa extensión de sus resultados.

La Figura 1 muestra una tosca medida de asociación entre acervo de infraestructura y crecimiento económico. La mayoría de los países de América Latina se encuentran por debajo de la línea de regresión ajustada a este conjunto de puntos. Esto implica que típicamente esos países muestran promedios de crecimiento en el producto por trabajador inferiores a los de países con similar crecimiento del acervo de infraestructura por trabajador, mientras que los países del este asiático exhiben niveles crecimientos superiores a los del promedio señalado por la línea de regresión. Una de las hipótesis de esta investigación es que esa diferencia puede atribuirse a factores institucionales (fragilidad política, facilidad para hacer negocios, libertad económica, percepción de corrupción, etc.) que condicionan la efectividad del gasto en infraestructura. 


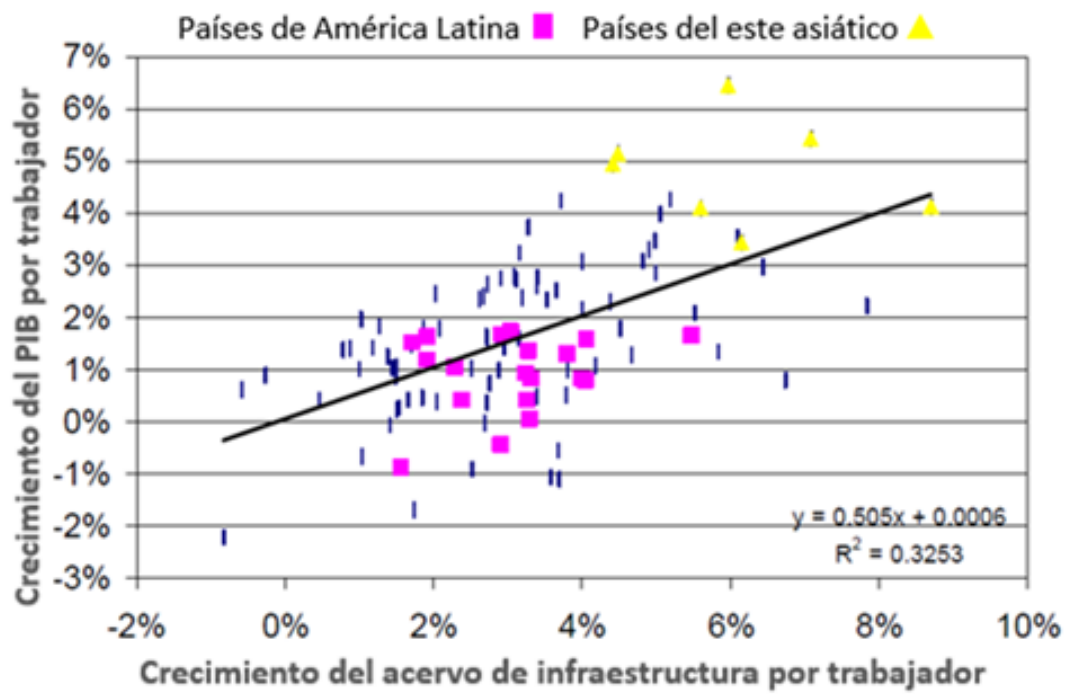

Figura 1. Crecimiento del acervo de infraestructura y producto por trabajador. Promedio 1960-1997. Fuente: Calderón y Servén (2002).

Esta investigación pretende no solo constituir una prueba de robustez de los resultados de Calderón y Servén (2002 y 2004), mediante la actualización del periodo de análisis, sino además extender ese estudio explorando la existencia de factores institucionales que condicionan la efectividad de la inversión en infraestructura.

El resto del documento está organizado de la siguiente forma: en la sección 2 se describen detalles metodológicos de la investigación. Los resultados de las estimaciones se resumen en la sección 3. La sección 4 se dedica a ejercicios de contraste de hipótesis aplicados al caso de Costa Rica, con los que se trata de dimensionar el orden de magnitud de las estimaciones obtenidas. Por último, la quinta sección ofrece las conclusiones.

\section{Metodología y modelo econométrico}

El enfoque metodológico se basa en Calderón y Servén (2002 y 2004). Se parte de una función de producción Cobb-Douglas linearizada y aumentada con capital de infraestructura como (1):

$$
y=\alpha k+\beta h+(1-\alpha-\beta-\gamma) l+\gamma z+u
$$

Donde $y$ es producción, $k$ es acervo de capital físico que no es de infraestructura, $h$ es capital humano, $l$ cantidad de trabajo, y $z$ capital de infraestructura. Como es usual, $u$ representa la parte

44

Manfred Esquivel Monge y Kerry Loaiza Marín

\section{(c) (i) (9) (2)}

Revista Economía y Sociedad by Universidad Nacional is licensed under a CreativeCommons Reconocimiento-NoComercial- 
del producto no explicada por factores de producción. Las variables son expresadas en logaritmos y se asume retornos constantes de escala.

El parámetro $\gamma$ capta la elasticidad del producto respecto a la infraestructura dados los demás factores. Nótese que en la información sobre formación bruta de capital de las cuentas nacionales el capital de infraestructura será doblemente contabilizado en la ecuación (1), primero como parte de $k$ y, separadamente, de $z$. Así que, según lo señala Canning (1999), el parámetro $\gamma$ captura la extensión sobre la cual la productividad de la infraestructura excede (si $\gamma>0$ ) o no (si $\gamma<0$ ), la productividad del capital que no es de infraestructura.

Si se considera la medida del acervo de capital $(k)$ como una suma ponderada de infraestructura y otros activos físicos, cuyas ponderaciones corresponden a sus respectivos precios relativos, esta puede expresarse de la siguiente forma:

$$
k \approx \frac{\widetilde{K}}{\widetilde{K}+p_{z} Z} \tilde{k}+\frac{p_{z} Z}{\widetilde{K}+p_{z} Z} Z
$$

Las mayúsculas denotan valores nominales; $\tilde{k}$ es capital físico que no es de infraestructura; $p_{z}$ es el precio relativo del capital de infraestructura en términos del capital que no es de infraestructura. Combinando (1) y (2) la elasticidad del producto respecto a infraestructura sería:

$$
\frac{\partial y}{\partial z}=\gamma+\theta \alpha \equiv \eta_{z}
$$

Donde

$$
\theta \equiv \frac{p_{z} Z}{\widetilde{K}+p_{z} Z}
$$

$\theta$ es la fracción que representa la infraestructura en el acervo total de capital físico ${ }^{4}$. En la práctica $\theta$ es pequeña, por lo que la diferencia entre $\eta_{z}$ y la estimación de $\gamma$ también lo es.

\subsection{Aproximación empírica}

Para la estimación el modelo (1) se reescribe en términos "por trabajador" tomando la diferencia logarítmica respecto a la fuerza laboral y, dado que se utiliza un panel datos, las especificaciones base tienen la siguiente forma general:

$$
y_{i t}-l_{i t}=a_{i}+b_{t}+\alpha\left(k_{i t}-l_{i t}\right)+\beta\left(h_{i t}-l_{i t}\right)+\gamma\left(z_{i t}-l_{i t}\right)+\varepsilon_{i t}
$$

\footnotetext{
${ }^{4}$ Estas expresiones involucran aproximaciones log-lineales alrededor de un punto arbitrario (por ejemplo, la media muestral).
} 
Los subíndices $i$ y $t$ denotan países y años; $a_{i}$ y $b_{t}$ capturan factores de productividad específicos por país y en el tiempo; y $\varepsilon_{i t}$ es la perturbación aleatoria no correlacionada a lo largo de $i$ y $t$.

El modelo (5) es el utilizado por Calderón y Servén (2002 y 2004). Acá se añaden controles por calidad institucional, quedando la siguiente forma general:

$$
y_{i t}^{*}=a_{i}+b_{t}+\alpha k_{i t}^{*}+\beta h_{i t}^{*}+\gamma z_{i t}^{*}+\rho d_{i t}+\rho_{2}\left(d_{i t} z_{i t}^{*}\right)+\varepsilon_{i t}
$$

Las variables con $\left({ }^{*}\right)$ están en términos por trabajador y $d_{i t}$ representa factores institucionales que varían por país y en el tiempo.

La versión dinámica del modelo de datos de panel sería la siguiente:

$$
y_{i t}^{*}-y_{i t-1}^{*}=a_{i}+b_{t}+\delta y_{i t-1}^{*}+\pi g_{i t}^{*}+\gamma z_{i t}^{*}+\rho d_{i t}+\rho_{2}\left(d_{i t} z_{i t}^{*}\right)+\varepsilon_{i t}
$$

Donde $g_{i t}^{*}$ es una matriz que contiene determinantes estándar de crecimiento en términos per cápita $\left(k_{i t}^{*}, h_{i t}^{*}\right)$. La utilización de una estructura de datos de panel para estimar modelos como (7) genera algunas dificultades econométricas. Dado que los componentes de $g_{i t}^{*}$ y $z_{i t}^{*}$ son determinados conjuntamente con $y_{i t}$, la estimación de (7) mediante mínimos cuadrados ordinarios padecerá endogeneidad. Además, la variable explicativa que induce dinámica $\left(y_{i t-1}\right)$, también es endógena si existen factores no observables fijos en el tiempo.

El uso de variables instrumentales (VI) solucionaría esta complicación, pero al no ser fácilmente identificables suficientes $\mathrm{VI}$, es posible apoyarse en instrumentos internos (rezagos de las explicativas) en línea con Arellano y Bond (1991).

Si efectivamente existen factores no observables fijos en el tiempo, es posible que $E\left[X_{i t-s}, A_{i}\right] \neq$ 0 , con lo cual retardos de las explicativas no serían instrumentos válidos. Esta complicación se supera tomando la primera diferencia de (7):

$$
\left(y_{i t}^{*}-y_{i t-1}^{*}\right)=b_{t}-b_{t-1}+(\delta+1)\left(y_{i t-1}^{*}-y_{i t-2}^{*}\right)+\left(X_{i t}-X_{i t-1}\right) B+\varepsilon_{i t}-\varepsilon_{i t-1}
$$

Donde se ha concatenado por filas las matrices $g_{i t}^{*}, z_{i t}^{*}$ y $d_{i t}$ resultado en la matriz $X_{i t}$. De acuerdo con Arellano y Bond (1991), si $\varepsilon_{i t}$ no está autocorrelacionada y las variables explicativas en $X_{i t}$ son débilmente exógenas, valores rezagados de las variables endógenas proveen instrumentos válidos. Tales supuestos definen las siguientes condiciones de momentos en la estimación del modelo diferencias:

i. $\quad E\left[y_{i t-s}\left(\varepsilon_{i t}-\varepsilon_{i t-1}\right)\right]=0$ para $s \geq 2$ y $t=3,4, \ldots T$

46

Manfred Esquivel Monge y Kerry Loaiza Marín

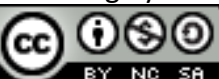

Revista Economía y Sociedad by Universidad Nacional is licensed under a CreativeCommons Reconocimiento-NoComercial- 
ii. $E\left[X_{i t-s}\left(\varepsilon_{i t}-\varepsilon_{i t-1}\right)\right]=0$ para $s \geq 2$ y $t=3,4, \ldots T$

Según Blundell y Bond (1998), cuando explicativas de la regresión en diferencias (8) exhiben persistencia, rezagos de endógenas pueden ser instrumentos débiles, resultando en alta variancia de los estimadores e incluso grandes sesgos asintóticos. Debido a esto Calderón y Servén (2002 y 2004) estiman un sistema que combina las regresiones en diferencia y en niveles en línea con Arellano y Bover (1995) y Blundell y Bond (1998).

En ese sistema de ecuaciones los instrumentos de la regresión en diferencia son los rezagos de las variables endógenas, y los de la regresión en niveles son rezagos de las diferencias de las variables endógenas correspondientes. Si no hay correlación entre las diferencias de esas variables y los efectos inobservables fijos en el tiempo, tales instrumentos son apropiados. De lo anterior se derivan las siguientes condiciones de momentos para las regresiones en niveles:

$$
\begin{array}{ll}
\text { iii. } & E\left[\left(y_{i t-1}-y_{i t-2}\right)\left(A_{i}+\varepsilon_{i t}\right)\right]=0 \\
\text { iv. } & E\left[\left(X_{i t-1}-X_{i t-2}\right)\left(A_{i}+\varepsilon_{i t}\right)\right]=0
\end{array}
$$

Las condiciones de momentos i, ii, iii y iv permiten aplicar GMM para generar estimaciones consistentes de los parámetros y su matriz de variancias y covariancias asintótica. Estas estarían dadas por:

$$
\begin{aligned}
& \widehat{\Upsilon}=\left(\bar{X}^{\prime} W \widehat{\Omega}^{-1} W^{\prime} \bar{X}\right)^{-1} \bar{X}^{\prime} W \widehat{\Omega}^{-1} W^{\prime} \bar{y} \\
& \operatorname{Asy} \operatorname{Var}(\widehat{\Upsilon})=\left(\bar{X}^{\prime} W \widehat{\Omega}^{-1} W^{\prime} \bar{X}\right)^{-1}
\end{aligned}
$$

Donde: $\bar{y}$ es la variable dependiente apilada primero en diferencias y luego en niveles; $\bar{X}$ es la matriz de variables explicativas $\left(y_{i t-1} X_{i t} d_{i t}\right)$, apilada primero en diferencias y luego en niveles; $W$ es la matriz de instrumentos derivada de las condiciones de momentos y $\hat{\Omega}$ es un estimador consistente de la matriz Var-Cov de las condiciones de momentos.

Para someter a prueba la validez de las condiciones de momentos, Arellano y Bond (1991) proponen una prueba de restricciones sobreidentificadas, en línea con Sargan (1958) y Hansen (1982). Además, se aplicó otra prueba, propuesta por Arellano y Bover (1995), que examina la hipótesis $\left(H_{0}\right)$ de que los errores $\varepsilon_{i t}$ no están serialmente correlacionados. El no rechazo $H_{0}$ constituye evidencia en favor de la estimación GMM. En la estimación que se propone se somete a prueba si los residuos de la regresión en diferencias muestran correlación serial de segundo orden. 


\subsection{Datos}

Salvo el Índice de Percepción de Corrupción (IPC), el de Libertad Económica (ILE) y el de Facilidad para Abrir Negocios (IFN), todas las variables se obtuvieron del Banco Mundial ${ }^{5}$. Los datos de fuerza laboral provienen del Banco Mundial para el periodo de 1990 a 2014 y para extender las series hasta 1960 se interpolaron con la base Summer-Heston.

Calderón y Servén (2002 y 2004) utilizan un panel de 121 países con información anual del periodo 1960-2000. Nuestra muestra abarca hasta el año 2014 y utiliza 213 países. Las variables utilizadas son las siguientes:

- PIB a precios constantes.

- Formación bruta de capital (FBK).

- FBK del sector público (por diferencia a partir del porcentaje del PIB que representa la FBK del sector privado).

- Fuerza laboral. Población mayor de 15 años que cumple la definición de población económicamente activa de la Organización Mundial del Trabajo.

- Consumo total de generación de energía eléctrica como proxy de acervo de infraestructura eléctrica.

- Kilómetros de ruta de línea férrea en uso como proxy de infraestructura de transporte ${ }^{6}$.

- Población total. Su logaritmo es utilizado como VI.

- Índice de Libertad Económica7. Es el promedio simple de la puntuación de cada país en las siguientes áreas: tamaño del estado, sistema judicial y derechos de propiedad, solidez monetaria, libertad de comercio internacional y regulación económica.

- Índice de percepción de la corrupción ${ }^{8}$. Mide la percepción de corrupción en el gobierno que tiene el empresariado.

- Índice de Facilidad de Apertura de Negocio ${ }^{9}$. Se mide en una escala de 0 a 100, donde 0 representa el peor desempeño y 100 el mejor.

- Infraestructura en telecomunicaciones. Suma del número de subscripciones activas de telefonía fija y celular.

- Capital humano. Corresponde a la multiplicación del porcentaje bruto de matrícula en educación secundaria por la razón de población entre 15 a 65 años a población de 0 a 14 años. Esta variable puede interpretarse como la fracción del tiempo que un trabajador

\footnotetext{
${ }^{5}$ http://databank.worldbank.org/data/reports.aspx?source=2\&Topic $=3$

${ }^{6}$ La International Road Federation colecta información sobre kilómetro y densidad de carreteras; sin embargo, el costo para accederla es elevado y no fue posible obtenerla.

${ }^{7}$ http://www.freetheworld.com/release.html

8 http://www.transparency.org/research/cpi/overview

9 http://www.doingbusiness.org/data
}

48

Manfred Esquivel Monge y Kerry Loaiza Marín 
dedica a aumentar su acervo de capital humano relativo al total de tiempo que podría emplear laborando ${ }^{10}$.

Las variables enumeradas de la i a la iii son en dólares estadounidenses a precios del 2005. Tanto la libertad económica (LE) como la percepción de corrupción (PC) se miden en una escala 0 al 10, donde 0 es la peor calificación y 10 la mejor.

\subsection{Proceso de estimación}

A partir de una estructura de datos de panel, se obtienen estimadores GMM en primeras diferencias utilizando como instrumentos el valor actual y tres rezagos del logaritmo de la población, más los segundos rezagos de las variables explicativas. Se aplicó una estimación en dos etapas para lidiar con problemas de heterocedasticidad comunes, usuales en este tipo de modelos.

Con el fin de corroborar si los factores institucionales condicionan el impacto de la infraestructura sobre el crecimiento, se crearon variables dicotómicas del IPC, ILE IFN. Estas se construyeron dividiendo la muestra en dos partes de igual tamaño, estableciendo la mediana de la distribución como umbral para la clasificación de países ${ }^{11}$. La mediana considera todos los años y países del estudio. Se asigna un uno a países cuyo índice es igual o mayor a la mediana y cero para aquellos con un valor menor. Estas variables indicadoras entran de forma aditiva y multiplicativa en las estimaciones.

\section{Resultados}

La Tabla 1 muestra un resumen de los resultados más relevantes de la investigación. La infraestructura impacta el crecimiento con coeficientes que son estadísticamente significativos y de entre 0,23 y 0,29 en magnitud. Dado que la estimación es en primeras diferencias, estas magnitudes indican que 1 punto porcentual (p.p.) adicional de crecimiento del capital de infraestructura por trabajador se asocia con un crecimiento entre 0,23 y 0,29 p.p. mayor del PIB por trabajador. Calderón y Servén (2002) estiman este efecto en alrededor de 0,36 p.p. La especificación 2 incluye inversión en infraestructura solo del sector público, pero por la limitada extensión de las series, no fue posible controlar por factores institucionales en ese contexto.

El efecto de los factores institucionales no solo se da sobre el nivel de crecimiento, sino también sobre la efectividad de la infraestructura para generar crecimiento. Las especificaciones 3 a 8 contienen resultados que permiten analizar estos impactos. En 3 y 4 se controló por el ILE y el IPC

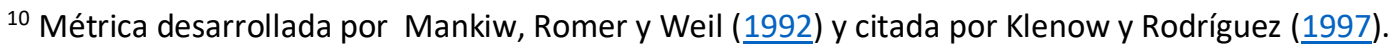

11 Se efectuaron estimaciones con dicotómicas construidas con umbrales en los percentiles 25 y 75 . Los resultados fueron muy similares.
}

Manfred Esquivel Monge y Kerry Loaiza Marín

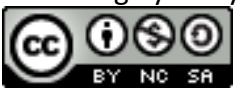

Revista Economía y Sociedad by Universidad Nacional is licensed under a CreativeCommons Reconocimiento-NoComercial- 
en su nivel original de forma separada y en 7 de forma conjunta. Los casos 5 y 6 corresponden, respectivamente, al control con las versiones dicotómicas del ILE y el IPC de forma separada. En el caso 8 se controla por la versión dicotómica de estas explicativas de forma conjunta e interactuando con infraestructura.

Según la especificación 7, se espera que un país que mejore su IPC en un punto obtenga un crecimiento del PIB por trabajador 1,5 p.p. mayor durante el mismo periodo. Una mejora de igual cuantía del ILE se asocia con un crecimiento del PIB por trabajador 3,1 p.p. más elevado dos periodos en el futuro.

Según el modelo 8, en países con menos PC cada p.p. adicional de crecimiento en infraestructura genera 0,0036 p.p. más de crecimiento. La LE condiciona en una magnitud similar la efectividad de la infraestructura sobre el crecimiento (coeficiente asociado de 0,0031), con la salvedad de que el efecto se da luego de dos años. Ambas pendientes diferenciales son significativas al $1 \%$ y $7 \%$, respectivamente ${ }^{12}$.

El efecto rezagado que muestra el ILE, y que no se da con el IPC, podría atribuirse a que este último se calcula con base en opiniones del sector empresarial que podrían manifestarse de forma más inmediata, mientras que el ILE se basa en indicadores prácticos de mejoras en el marco institucional que propician mejoras en transacciones y toma de decisiones, por lo que su impacto podría tomar más tiempo.

Entre los tres tipos de infraestructura por los cuales se controla separadamente, la eléctrica tiene mayor efecto sobre el crecimiento; 1 p.p. adicional de crecimiento en capacidad de producción eléctrica se asocia con entre 0,11 y 0,21 p.p. más de crecimiento. El efecto de la infraestructura de telecomunicaciones se estima entre 0,035 y 0,06 p.p. El acervo de líneas férreas no resultó estadísticamente significativo.

El coeficiente asociado a capital humano resultó positivo, significativo en todas las especificaciones y de magnitud es cercana a 0,0008. Según Klenow y Rodríguez (1997), la forma en que se mide este capital es la fracción del tiempo que un trabajador dedica a aumentar su acervo de conocimientos relativo al total de tiempo que podría emplear laborando. Entonces la magnitud estimada del coeficiente implicaría que, si la fuerza laboral incrementa en una unidad esa fracción de tiempo, se espera que el crecimiento del PIB por trabajador sea 0,08 p.p. mayor.

\footnotetext{
12 En la Tabla 4 del apéndice se muestran los resultados de estimaciones utilizando el índice Starting a Business como indicador de factores institucionales. Estos son congruentes con lo que se muestra en la Tabla 1 (efecto positivo y significativo del indicador institucional tanto en nivel como interactuando con infraestructura). La corta extensión del indicador "Starting a Business" limita el número de observaciones.
}

50

Manfred Esquivel Monge y Kerry Loaiza Marín

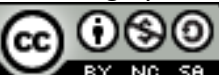

Revista Economía y Sociedad by Universidad Nacional is licensed under a CreativeCommons Reconocimiento-NoComercial- 
Tabla 1.

Resultados de estimación GMM. Variable dependiente: $\Delta \log (P I B /)$

\begin{tabular}{|c|c|c|c|c|c|c|c|c|c|}
\hline & \multicolumn{8}{|c|}{ Especificaciones alternativas* } \\
\hline & & 1 & 2 & 3 & 4 & 5 & 6 & 7 & 8 \\
\hline \multirow{2}{*}{ Capital infraestructura } & & 0.2948 & & 0.2891 & 0.2362 & 0.2819 & 0.2436 & 0.233 & 0.2346 \\
\hline & & $(0,000)$ & & $(0,000)$ & $(0,000)$ & $(0,000)$ & $(0,000)$ & $(0,000)$ & $(0,000)$ \\
\hline \multirow{2}{*}{\multicolumn{2}{|c|}{ Capital infraestructura sector público }} & & 0.1221 & & & & & & \\
\hline & & & $(0,000)$ & & & & & & \\
\hline \multirow{2}{*}{\multicolumn{2}{|c|}{ Telecomunicaciones }} & 0.0508 & 0.0478 & 0.0545 & 0.0352 & 0.0595 & 0.0372 & 0.0403 & 0.0463 \\
\hline & & $(0,004)$ & $(0,014)$ & $(0,008)$ & $(0,034)$ & $(0,001)$ & $(0,017)$ & $(0,042)$ & $(0,004)$ \\
\hline \multirow{2}{*}{\multicolumn{2}{|c|}{ Generación eléctrica }} & 0.2118 & 0.2149 & 0.1155 & 0.2199 & 0.1147 & 0.2024 & 0.2035 & 0.1937 \\
\hline & & $(0,000)$ & $(0,001)$ & $(0,054)$ & $(0,005)$ & $(0,039)$ & $(0,003)$ & $(0,003)$ & $(0,005)$ \\
\hline \multirow{2}{*}{\multicolumn{2}{|c|}{ Líneas de tren }} & 0.0907 & 0.2039 & -0.0053 & -0.0154 & 0.0021 & -0.0135 & -0.0132 & -0.013 \\
\hline & & $(0,044)$ & $(0,001)$ & $(0,824)$ & $(0,364)$ & $(0,932)$ & $(0,457)$ & $(0,498)$ & $(0,420)$ \\
\hline \multirow{2}{*}{\multicolumn{2}{|c|}{ Capital humano }} & 0.0008 & 0.0017 & 0.0009 & 0.0007 & 0.0008 & 0.0007 & 0.0007 & 0.0006 \\
\hline & & $(0,000)$ & $(0,000)$ & $(0,005)$ & $(0,013)$ & $(0,003)$ & $(0,007)$ & $(0,016)$ & $0,022)$ \\
\hline \multirow{2}{*}{\multicolumn{2}{|c|}{ Percepción corrupción }} & & & 0.0121 & & & & 0.0152 & \\
\hline & & & & $(0,033)$ & & & & $(0,087)$ & \\
\hline \multirow{2}{*}{\multicolumn{2}{|c|}{ Libertad económica (t-2) }} & & & & 0.0406 & & & 0.0311 & \\
\hline & & & & & $(0,008)$ & & & $(0,044)$ & \\
\hline \multirow{2}{*}{\multicolumn{2}{|c|}{$\begin{array}{l}\text { Dummy (percepción corrupción) * } \\
\text { capital infraestructura }\end{array}$}} & & & & & 0.0039 & & & 0.0036 \\
\hline & & & & & & $(0,002)$ & & & $(0,005)$ \\
\hline \multirow{2}{*}{\multicolumn{2}{|c|}{$\begin{array}{l}\text { Dummy libertad económica(t-2)] } \\
\text { capital infraestructura }\end{array}$}} & & & & & & 0.0033 & & 0.0031 \\
\hline & & & & & & & $(0,002)$ & & $(0,072)$ \\
\hline $\begin{array}{l}\text { Prueba de Wald de significancia } \\
\text { conjunta (valor } \mathrm{P} \text { ) }\end{array}$ & & 0.00 & 0.00 & 0.00 & 0.00 & 0.00 & 0.00 & 0.00 & 0.00 \\
\hline \multirow{2}{*}{ Test de autocorrelación (valor p) } & Primer orden & 0.36 & 0.09 & 0.00 & 0.04 & 0.04 & 0.05 & 0.04 & 0.61 \\
\hline & Segundo orden & 0.40 & 0.49 & 0.18 & 0.56 & 0.15 & 0.64 & 0.45 & 0.43 \\
\hline $\begin{array}{l}\text { Prueba de Sargan de restricciones } \\
\text { sobre identificadas (valor } \mathrm{P} \text { ) }\end{array}$ & & 1.00 & 1.00 & 1.00 & 1.00 & 1.00 & 1.00 & 1.00 & 1.00 \\
\hline No. Observaciones & & 1565 & 640 & 780 & 498 & 780 & 496 & 495 & 493 \\
\hline No. Países & & 96 & 53 & 82 & 67 & 82 & 67 & 67 & 67 \\
\hline
\end{tabular}

*Valores $\mathrm{P}$ robustos (Huber y White) entre paréntesis.

Fuente: elaboración propia

En términos de diagnóstico econométrico, en las ocho especificaciones las pruebas de Wald de significancia conjunta señalan evidencia de que la asociación entre la dependiente y las explicativas no es producto del azar o muestra particular utilizada. Según las pruebas de autocorrelación no existe evidencia que desfavorezca el uso de las condiciones de momentos empleadas. Las pruebas de restricciones sobreidentificadas validan que los instrumentos en exceso son exógenos.

Como complemento al resultado que señala un efecto significativo sobre la efectividad de la infraestructura tanto de la PC como de la LE, la Figura $\underline{2}$ y la Figura $\underline{3}$ posibilitan identificar qué países presentan mejores niveles en estos indicadores. 


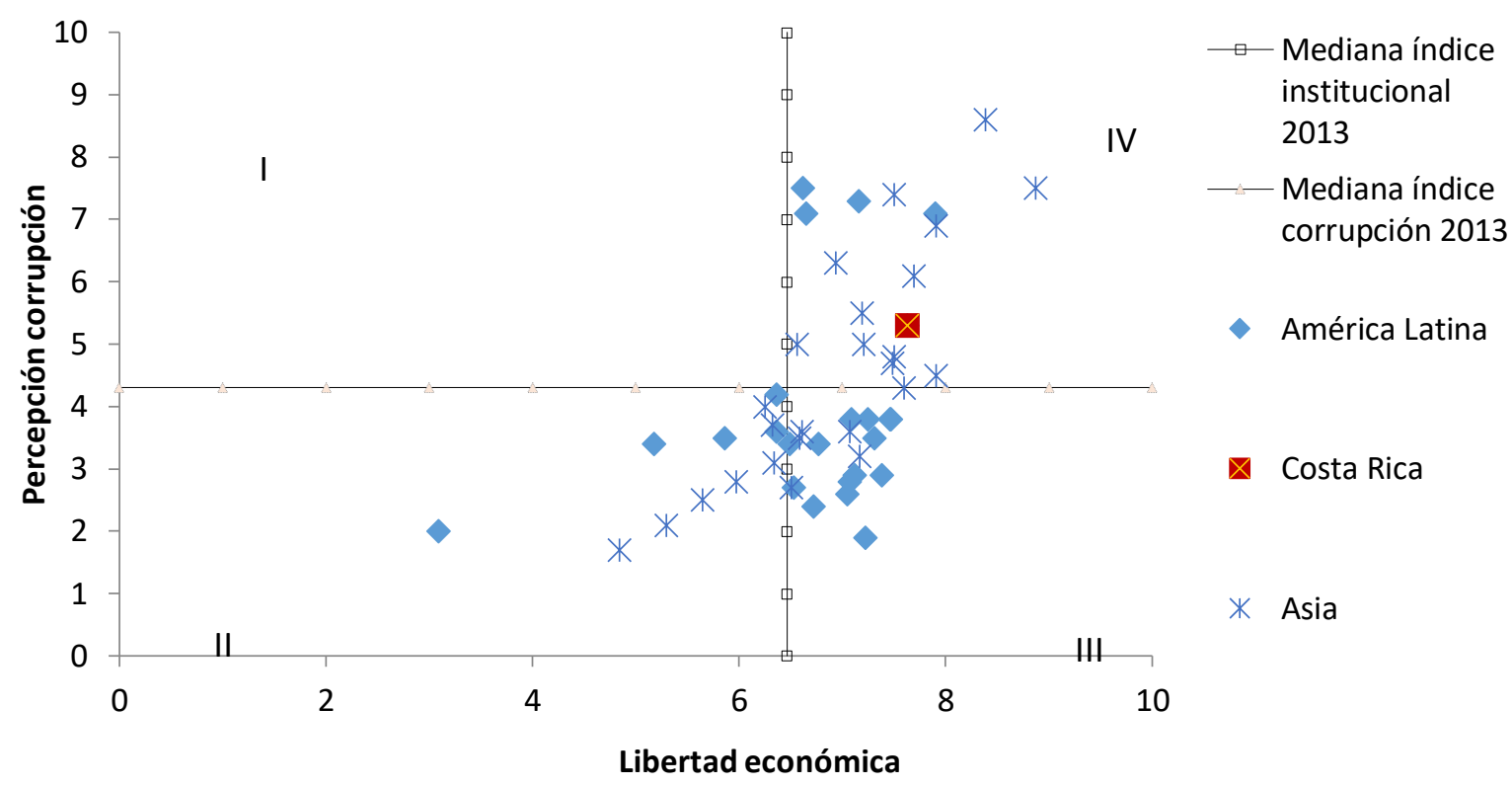

Figura 2. Índices de percepción de corrupción libertad económica por región, año 2013. Fuente: elaboración propia.

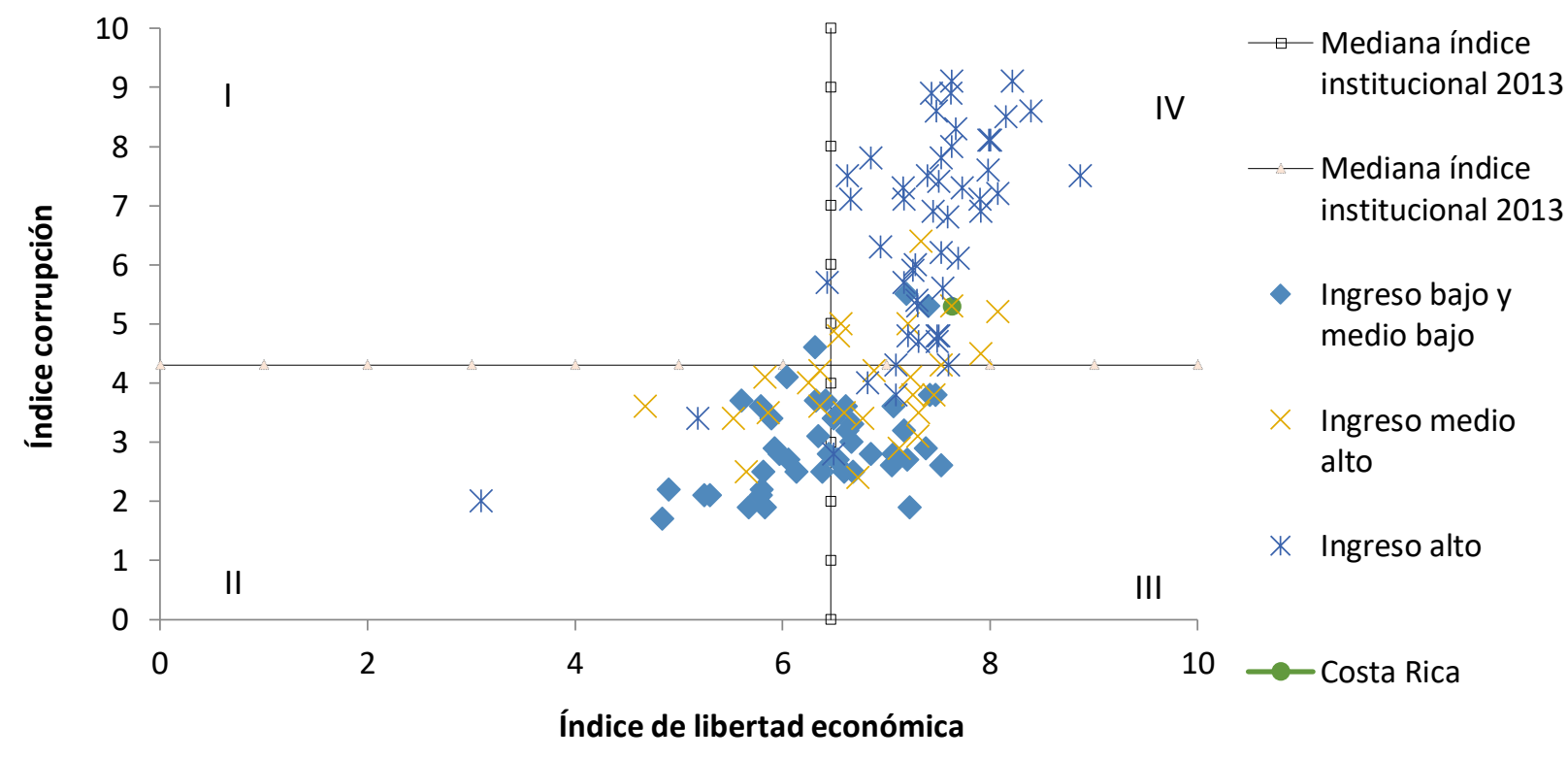

Figura 3. Índices de percepción de corrupción y libertad económica por nivel de ingreso, año 2013. Fuente: elaboración propia.

52 
En la Figura $2 \underline{2}$ diferencia entre países de América Latina y Asia. La mayoría de los países de América Latina se ubica en los cuadrantes II y III, que corresponden a niveles de PC peores al de la mediana de la distribución. Según los resultados econométricos, estos son países donde la PC resta eficacia (en términos de generar crecimiento) a la infraestructura. En cuanto a LE, la distribución entre países de América Latina y Asia es más simétrica. Costa Rica en se encuentra en el cuadrante IV, por encima de la mediana tanto en IPC como de ILE.

La Figura $\underline{3}$ distingue los países según su nivel de ingreso. Casi la totalidad de aquellos con ingreso alto se ubican en el cuadrante IV, con niveles del IPC y del ILE superiores a los de países de ingreso bajo y medio. Casi todos los países de ingreso bajo y medio bajo están en los cuadrantes II y III, lo cual disminuye el potencial generador de crecimiento de su infraestructura.

La definición de cuadrantes que se presenta en la Figura $\underline{2}$ y la Figura $\underline{3}$ sugiere la posibilidad de conocer el efecto conjunto del ILE y el IPC sobre la efectividad de la infraestructura para generar crecimiento. Para esto se modificaron las especificaciones econométricas para incluir una sola variable categórica que define 4 tipos de países: con favorable PC y baja LE (cuadrante I), con desfavorable PC y baja LE (cuadrante II), con desfavorable PC y alta LE (cuadrante III) y, finalmente, con favorable PC y alta LE (cuadrante IV). Fijando esta última como categoría de control, los resultados se muestran en la Tabla $\underline{2}$.

La magnitud y significancia de los coeficientes asociados a las variables indicadoras de infraestructura es similar a las ya comentadas de la Tabla 1 . Como es esperable, debido a que la categoría de control son países del cuadrante IV, tanto los interceptos como las pendientes diferenciales son negativos. Esto indica que tanto el crecimiento promedio como el efecto esperado de la infraestructura sobre el crecimiento son mayores en los países de la categoría de control.

Según la especificación 5, mientras en los países del cuadrante IV 1 p.p. más de crecimiento en el acervo de infraestructura se asocia con 0,27 p.p. más de crecimiento, en los países del cuadrante II generará cerca de 0,26 p.p. adicionales. Si bien la magnitud del impacto no es grande, es estadísticamente significativa.

La pendiente diferencial asociada a la dicotómica indicadora de cuadrante 1 es mayor que la asociada al cuadrante 3. Esto implica que el desempeño en LE impacta más que el desempeño en PC sobre la efectividad de la infraestructura para generar crecimiento.

La evidencia econométrica de las especificaciones 3 a 8 de la Tabla $\underline{1}$, y de la especificación 5 de la Tabla 2 , junto con las asociaciones entre los índices de PC y LE según región o nivel de ingreso, pueden explicar, al menos en parte, tanto las amplias diferencias en crecimiento entre regiones (Asia vs. América Latina), como el impacto positivo de una buena gestión institucional. 


\section{Contraste de hipótesis para Costa Rica}

Para dimensionar estos resultados, se efectuaron varios ejercicios de contraste de hipótesis aplicados a la economía costarricense. Estos consistieron en presentar un escenario alternativo de crecimiento del capital por trabajador y cuantificar su efecto sobre el crecimiento a partir de los coeficientes estimados.

Se realizaron dos tipos de ejercicio, en el primero asume un crecimiento adicional de 1 p.p. del capital de infraestructura por trabajador para cada año desde 1961 hasta 2014. En el segundo se supone el mismo incremento del escenario anterior, pero condicionando la senda de crecimiento a la ubicación del país en los cuadrantes I, II, III o IV (ver Tabla 2).

\section{Tabla 2.}

Resultados de estimación GMM. Variable dependiente: $\Delta \log (P I B / L)$

\begin{tabular}{|c|c|c|c|c|c|c|c|c|c|}
\hline & \multicolumn{8}{|c|}{ Especificaciones alternativas* } \\
\hline & & 1 & 2 & 3 & 4 & 5 & 6 & 7 & 8 \\
\hline \multirow{2}{*}{\multicolumn{2}{|c|}{ Capital infraestructura }} & 0.2697 & 0,2788 & 0,2768 & 0,2870 & 0,2708 & 0,2819 & 0,2712 & 0,2874 \\
\hline & & $(0,000)$ & $(0,000)$ & $(0,000)$ & $(0,000)$ & $(0,000)$ & $(0,000)$ & $(0,000)$ & $(0,000)$ \\
\hline \multirow{2}{*}{\multicolumn{2}{|c|}{ Telecomunicaciones }} & 0.0431 & 0,0382 & 0,0460 & 0,0466 & 0,0455 & 0,0369 & 0,0454 & 0,0453 \\
\hline & & $(0,008)$ & $(0,052)$ & $(0,028)$ & $(0,018)$ & $(0,008)$ & $(0,019)$ & $(0,001)$ & $(0,026)$ \\
\hline \multirow{2}{*}{\multicolumn{2}{|c|}{ Generación eléctrica }} & 0,1962 & 0,2230 & 0,2112 & 0,2105 & 0,2091 & 0,2204 & 0,2200 & 0,2191 \\
\hline & & $(0,002)$ & $(0,001)$ & $(0,000)$ & $(0,001)$ & $(0,001)$ & $(0,001)$ & $(0,001)$ & $(0,000)$ \\
\hline \multirow{2}{*}{\multicolumn{2}{|c|}{ Líneas de tren }} & $-0,0019$ & $-0,0069$ & $-0,0114$ & $-0,0028$ & $-0,0035$ & $-0,0064$ & $-0,0067$ & $-0,0058$ \\
\hline & & $(0,924)$ & $(0,740)$ & $(0,571)$ & $(0,903)$ & $(0,858)$ & $(0,808)$ & $(0,757)$ & $(0,844)$ \\
\hline \multirow{2}{*}{\multicolumn{2}{|c|}{ Capital humano }} & 0,0007 & 0,0008 & 0,0008 & 0.0007 & 0,0007 & 0,0008 & 0,0008 & 0,0007 \\
\hline & & $(0,019)$ & $(0,025)$ & $(0,015)$ & $(0,065)$ & $(0,025)$ & $(0,011)$ & $(0,012)$ & $(0,017)$ \\
\hline \multirow{2}{*}{\multicolumn{2}{|c|}{ Dummy cuadrante 1}} & $-0,0778$ & $-0,0176$ & & & & & & \\
\hline & & $\begin{array}{l}(0,042) \\
-0,0822\end{array}$ & $(0,53 /)$ & -0.0075 & & & & & \\
\hline \multicolumn{2}{|l|}{ Dummy cuadrante 2} & $\begin{array}{l}-0,0822 \\
(0,001)\end{array}$ & & $\begin{array}{l}-0,00 / 5 \\
(0,624)\end{array}$ & & & & & \\
\hline \multirow{2}{*}{\multicolumn{2}{|c|}{ Dummy cuadrante 3}} & $-0,0651$ & & & $-0,0300$ & & & & \\
\hline & & $(0,000)$ & & & $(0,073)$ & & & & \\
\hline \multirow{2}{*}{\multicolumn{2}{|c|}{$\begin{array}{l}\text { Interacción dummy cuadrante } 1 \text { y } \\
\text { capital infraestructura }\end{array}$}} & & & & & $-0,0058$ & $-0,0010$ & & \\
\hline & & & & & & $(0,045)$ & $(0,689)$ & & \\
\hline \multirow{2}{*}{\multicolumn{2}{|c|}{$\begin{array}{l}\text { Interacción dummy cuadrante } 2 \text { y } \\
\text { capital infraestructura }\end{array}$}} & & & & & $-0,0065$ & & $-0,0014$ & \\
\hline & & & & & & $(0,004)$ & & $(0,344)$ & \\
\hline \multirow{2}{*}{\multicolumn{2}{|c|}{$\begin{array}{l}\text { Interacción dummy cuadrante } 3 \text { y } \\
\text { capital infraestructura }\end{array}$}} & & & & & $-0,0049$ & & & $-0,0028$ \\
\hline & & & & & & $(0,000)$ & & & $(0,036)$ \\
\hline $\begin{array}{l}\text { Prueba de Wald de significancia } \\
\text { conjunta (valor } \mathrm{P} \text { ) }\end{array}$ & & 0 & 0 & 0 & 0 & 0 & 0 & 0 & 0 \\
\hline \multirow[t]{2}{*}{ Test de autocorrelación (valor p) } & Primer orden & 0,53 & 0,04 & 0,04 & 0,06 & 0,45 & 0,04 & 0,4 & 0,09 \\
\hline & Segundo orde & 0,54 & 0,23 & 0,14 & 0,36 & 0,48 & 0,25 & 0,10 & 0,44 \\
\hline $\begin{array}{l}\text { Prueba de Sargan de restricciones } \\
\text { sobre identificadas (valor } \mathrm{P} \text { ) }\end{array}$ & & 1 & 1 & 1 & 1 & 1 & 1 & 1 & 1 \\
\hline No. Observaciones & & 574 & 574 & 574 & 574 & 574 & 574 & 574 & 574 \\
\hline No. Países & & 67 & 67 & 67 & 67 & 67 & 67 & 67 & 67 \\
\hline
\end{tabular}

${ }^{*}$ Valores $\mathrm{P}$ robustos (Huber y White) entre paréntesis.

Fuente: elaboración propia

54

Manfred Esquivel Monge y Kerry Loaiza Marín

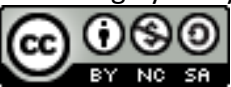

Revista Economía y Sociedad by Universidad Nacional is licensed under a CreativeCommons Reconocimiento-NoComercial- 
4.1. Impacto a largo plazo de 1 p.p. más de crecimiento de la infraestructura

La pregunta por responder es: ¿cuál es la diferencia entre el PIB observado y el de una serie hipotética estimada suponiendo un crecimiento del acervo de capital por trabajador 1 p.p. superior al que se presentó desde el año 1961? Tal supuesto genera una nueva serie de capital por trabajador (K/L). Según las estimaciones mostradas, se espera que esto añada 0,29 p.p. al crecimiento del PIB por trabajador (PIB/L) cada año. Asumiendo que la fuerza laboral no varía su evolución observada, esto genera una nueva serie PIB/L.

Los resultados (ver Figura 4) sugieren que ante tal escenario en 2014 el PIB habría sido 16,4\% superior al observado. Además, se estima que la proporción (en promedio por década) del capital a PIB se habría comportado según se ilustra en la Tabla $\underline{3}$.

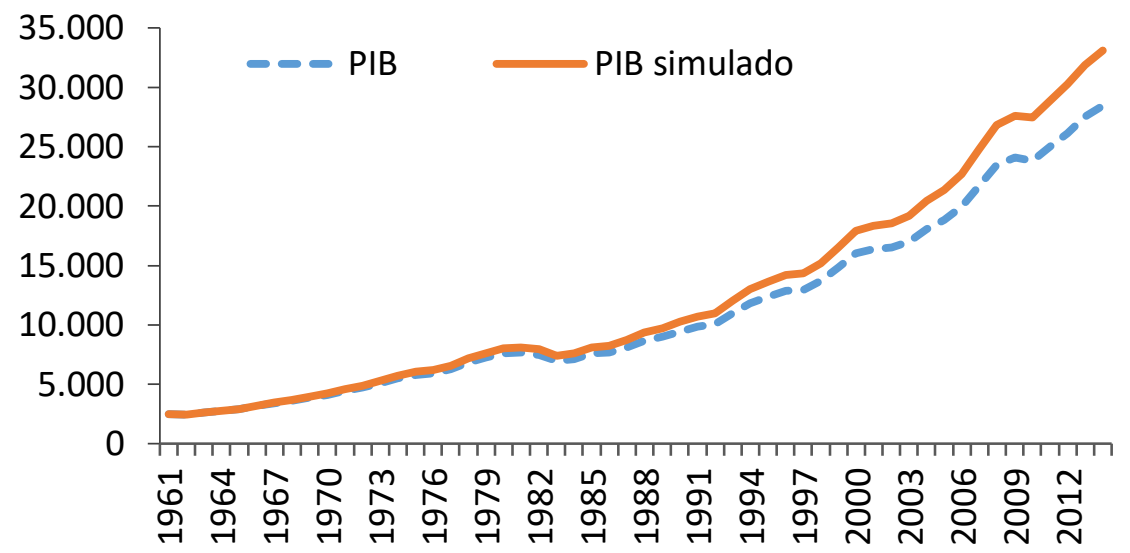

Figura 4. PIB observado y simulado. 1961 - 2014 (UA\$ de 2005). Fuente: elaboración propia.

\section{Tabla 3.}

Proporción promedio de K a PIB

\begin{tabular}{lll}
\hline & Observada & Simulada \\
\hline $1960-1969$ & 18 & 18 \\
$1970-1979$ & 23 & 25 \\
$1980-1989$ & 20 & 24 \\
$1990-1999$ & 19 & 24 \\
$2000-2013$ & 20 & 28 \\
\hline
\end{tabular}

Fuente: elaboración propia

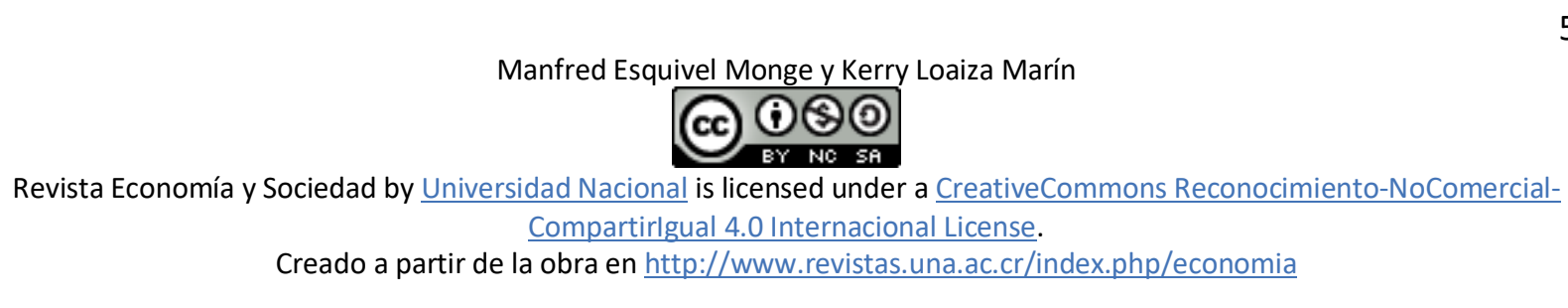




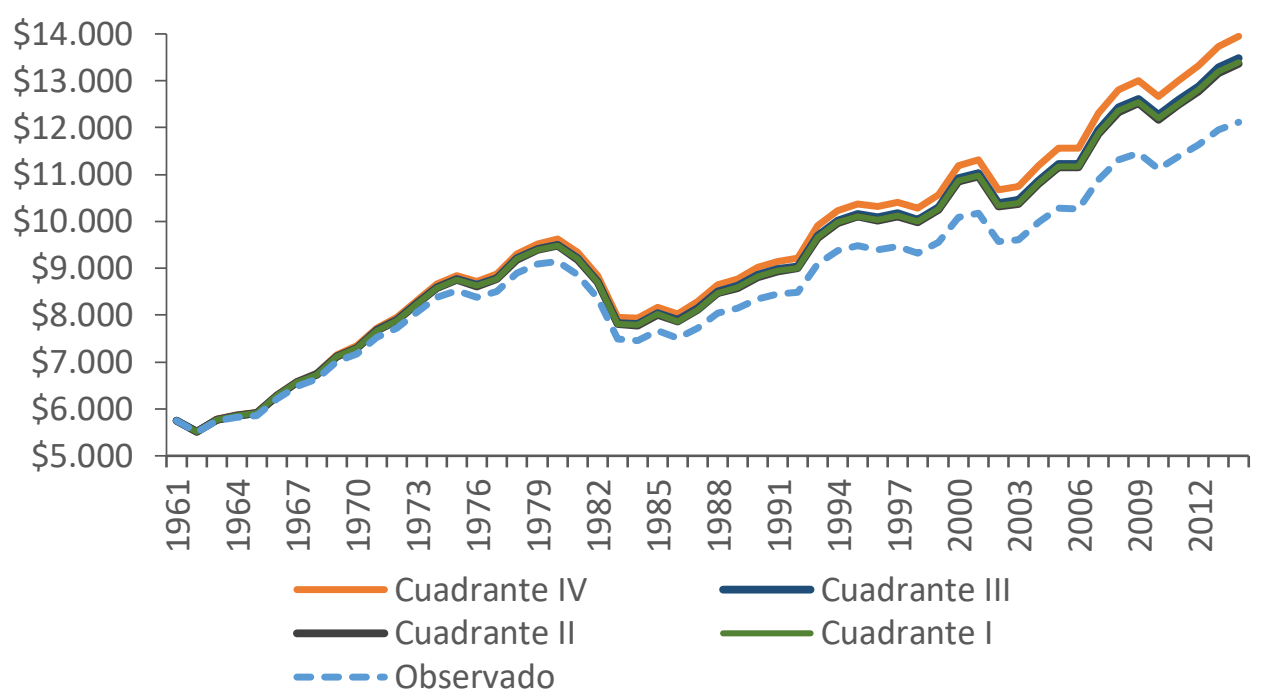

Figura 5. PIB por trabajador observado y simulado. 1961 - 2014 (UA\$ de 2005). Fuente: elaboración propia.

\subsection{Impacto a largo plazo de la infraestructura condicionando por factores institucionales}

Al igual que en ejercicio anterior, se supone un crecimiento 1 p.p. mayor de la infraestructura por trabajador entre 1961 y 2014 . Pero en este caso se condiciona el crecimiento por factores institucionales (ILE e IPC). Para esto se considera la especificación econométrica 1 de la Tabla $\underline{2}$ que incluye como variables explicativas las dicotómicas correspondientes a los cuadrantes que identifican a cada país según factores institucionales.

Asumiendo un crecimiento de 1 p.p. adicional del acervo de infraestructura y respecto a países del cuadrante IV, países en el cuadrante I y II presentan, cada año, un crecimiento por trabajador 0,0778 p.p. y 0,0822 p.p. inferior, respectivamente. Estos resultados se ilustran en las Figuras $\underline{5}$ y 6. La diferencia más cuantiosa respecto a la serie observada se presenta para el Cuadrante IV, seguida del Cuadrante III. Según estos resultados, si cada año desde 1961 hasta 2014 (53 años) el capital en infraestructura por trabajador hubiese crecido 1 p.p. adicional, en 2014 el PIB por trabajador habría sido superior al observado en cerca de EUA\$ 1.250 en los países de los cuadrantes I y II; aproximadamente EUA\$1.400 mayor en países del cuadrante III; y EUA\$1.830 mayor en los países del cuadrante IV (se trata de dólares constante del 2012). 


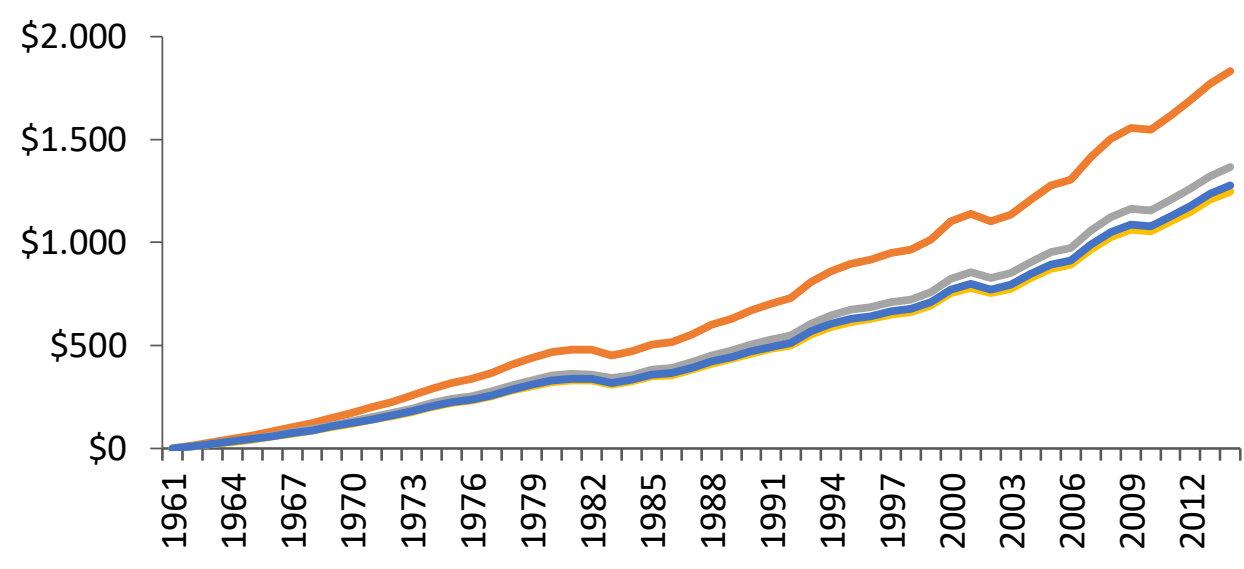

Cuadrante IV —Cuadrante III —Cuadrante II —Cuadrante I

Figura 6. Diferencia en el PIB por trabajador observado y simulado. 1961 - 2014 (EUA\$ de 2005). Fuente: elaboración propia

\section{Conclusiones}

Existe un efecto positivo y estadísticamente significativo de la infraestructura sobre el crecimiento económico. Este se estima entre 0,23 y 0,29 p.p. adicionales de crecimiento del producto per cápita por cada p.p. adicional de crecimiento del acervo de infraestructura. Estos resultados son coherentes con otras investigaciones en las que se utilizó un conjunto menor de información.

Mejoras en la calidad institucional impactan no solo el crecimiento sino la efectividad de la infraestructura para generar crecimiento. El impacto de mejoras en PC es contemporáneo, mientras que el de mejoras en LE se manifiesta dos años de rezago.

Los países con mayor LE y menor PC tienen, en promedio, no solo tasas de crecimiento superiores, sino que extraen mayor rédito de las inversiones que realizan en infraestructura. Un país con niveles de LE y PC superiores a la mediana de la distribución logra que cada p.p. de gasto en infraestructura se manifieste en 0,25 p.p. más de crecimiento contra solo 0,23 p.p. que logra un país cuyos indicadores lo ubican por debajo de la mediana.

La mayoría de países de América Latina tienen una PC que los ubica por debajo de la mediana de la distribución, mientras que una buena porción de países asiáticos está por sobre esa mediana. Casi la totalidad de países de ingreso alto tienen niveles de PC y de LE superiores a los de países de ingreso bajo y medio. Esta diferencia explicaría, al menos en parte, que países en América 
Latina $y$, en general, de ingreso medio o bajo, obtengan menor rédito de la inversión en infraestructura en términos de crecimiento.

Según los resultados del estudio, se estima que 1 p.p. adicional de crecimiento del acervo de capital de infraestructura por trabajador posibilitaría un PIB por trabajador cerca de $16 \%$ superior luego de 53 años. Países con mejor desempeño en libertad económica y menor precepción de corrupción obtendrían una mejoría más importante, en términos de crecimiento, ante este crecimiento adicional del acervo de infraestructura.

Según FMI (2014) y puesto que existe amplia documentación que señala un estancamiento en la inversión pública (ver Arce, Céspedes y Jiménez, 2006; Castro y Porras, 2009; Mesalles, 2010), en Costa Rica el crecimiento económico entre el año 2014 y el 2016 ha sido inferior al potencial estimado para la economía costarricense (4,4\%); y que durante este lapso de tiempo la tasa de desempleo abierto no ha bajado de $8,3 \%$, los resultados de este trabajo respaldan políticas que conlleven dar un impulso a la inversión pública en infraestructura, sin que sea esperable un deterioro importante en las finanzas públicas. Además, existe la posibilidad de potenciar el impacto de esa política expansiva mejorando los aspectos que se toman en cuenta para construir el índice de LE y disminuyendo la PC en el país.

\section{Referencias}

Arce, G., Céspedes, V. H., y Jiménez, R. (2006). La pobreza en Costa Rica 1987-2004. En V.H. Céspedes y R. Jiménez. Pobreza en Costa Rica. San José: Academia de Centroamérica. Recuperado http://enlaceacademico.ucr.ac.cr/sites/default/files/publicaciones/Librojornada3.pdf

Arellano, M. y Bond, S. (1991). Some Tests of Specification for Panel Data: Monte Carlo Evidence and an Application to Employment Equations. Review of Economic Studies 58(2), 277297. doi: http://dx.doi.org/10.2307/2297968

Arellano, M. y Bover, O. (1995). Another look at the Instrumental Variable Estimation of ErrorComponent Models. Journal of Econometrics 68(1), 29-51. doi: http://dx.doi.org/10.1016/0304-4076(94)01642-d

Aschauer, D. (1989). Is Public Expenditure Productive? Journal of Monetary Economics. 23(2), 177200. doi: http://dx.doi.org/10.1016/0304-3932(89)90047-0

Auerbach, A. y Gorodnichencko, Y. (2011). Fiscal Multipliers in Recession and Expansion. NBER Working Paper 17447. doi: http://dx.doi.org/10.3386/w17447

58

Manfred Esquivel Monge y Kerry Loaiza Marín

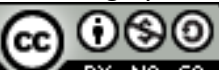

Revista Economía y Sociedad by Universidad Nacional is licensed under a CreativeCommons Reconocimiento-NoComercial- 
Auerbach, A. y Gorodnichencko, Y. (2012). Measuring the Output Responses to Fiscal Policy. American Economic Journal: Economic Policy 4 (2), 1-27. doi: http://dx.doi.org/10.1257/pol.4.2.1

Blundell, R. y Bond, R. (1998). Initial Conditions and Moment Restrictions in Dynamic Panel Data Models. Journal of Econometrics 87(1), 115-143. doi: http://dx.doi.org/10.1016/s03044076(98)00009-8

Calderón, C. y Servén, L. (2002). The Output Cost of Latin America's Infrastructure GAP. Working Paper $N^{\circ}$ 186. Recuperado de http://www.bcentral.cl/web/guest/-/the-output-cost-oflatin-america-s-infrastructure-g-2

Calderón, C. y Servén, L. (2004). The effects of infrastructure development on growth and income distribution. Policy Research Working Papers. doi: http://dx.doi.org/10.1596/1813$\underline{9450-3400}$

Calderón, C., Easterly, W. y Servén, L. (2003). Infrastructure Compression and Public Sector Solvency in Latin America. En W. Easterly, y L., Servén (eds.). The Limits of Stabilization: Infrastructure, Public Deficits and Growth in Latin America, 95-118. doi: http://dx.doi.org/10.1596/978-0-8213-5489-6

Canning, D. (1999). Infrastructure's contribution to aggregate output. World Bank Policy Research Discussion Paper 2246. doi: http://dx.doi.org/10.1596/1813-9450-2246

Castro, R. y Porras, J. A. (2009). Infraestructura y desarrollo económico en Costa Rica. En L. Mesalles, L. y O. Céspedes (eds.). Costa Rica 2008: Obstáculos al crecimiento económico. Recuperado de https://www.academiaca.or.cr/wpcontent/uploads/2017/05/Libroanual_conportda_09.pdf

Celis, R. (2007). Costa Rica: Identificación de la combinación de inversiones públicas más apropiada durante el periodo de transición hacia la entrada en vigencia del CAFTA. Costa Rica. Proyecto de cooperación técnica CEPAL/BID/IFPRI. Recuperado de https://www.cepal.org/publicaciones/xml/6/32316/costa_rica_1.pdf

Ferreira, F. (1995). Roads to Equality: Wealth Distribution Dynamics with Public-Private Capital Complementarity. LSE Discussion Paper TE/95/286. http://eprints.Ise.ac.uk/19368/1/Roads to Equality Wealth Distribution Dynamics w ith Public-Private Capital Complementarity.pdf 
Fondo Monetario Internacional. (2014). World Economic Outlook, October 2014: Legacies, Clouds and Uncertainties. doi: http://dx.doi.org/10.5089/9781484372265.081

Hansen, L. P. (1982). Large Sample Properties of Generalized Method of Moments Estimators. Econométrica 50 (4), 1029-1054. doi: http://dx.doi.org/10.2307/1912775

Klenow, P. y Rodríguez, A. (1997). The Neoclassical Revival in Growth Economics: Has It Gone Too Far? NBER Macroeconomics Annual 1997, 12, 73-103. doi: http://dx.doi.org/10.2307/3585220

Mankiw, N. G., Romer, D. y Weil, D. N. (1992). A contribution to the empirics of economic growth. Quaterly Journal of Economics 107 (2), 407-437. doi: http://dx.doi.org/10.2307/2118477

Mesalles, L. (2010). El proceso de políticas en el sector infraestructura de Costa Rica: actores, arenas e intercambios. San José, Costa Rica: Mimeo.

Mesalles, L. y Celis, R. (2011). Más y mejor infraestructura de transporte para reducir la pobreza rural en Costa Rica. En Fundação Konrad Adenauer. Inversión en infraestructura pública y reducción de la pobreza en América Latina. Recuperado de http://www.kas.de/wf/doc/kas 29022-1522-4-30.pdf?111114153158

Sargan, J. D. (1958). The Estimation of Economic Relationships Using Instrumental Variables. Econométrica 26 (3), 393-415. doi: http://dx.doi.org/10.2307/1907619

60 


\section{Apéndice}

Tabla 4.

Resultados de estimación GMM. Variable dependiente: $\Delta \log (P I B / L)$

\begin{tabular}{|c|c|c|c|c|}
\hline & \multicolumn{3}{|c|}{ Especificaciones alternativas* } \\
\hline & & 1 & 2 & 3 \\
\hline \multirow{2}{*}{\multicolumn{2}{|c|}{ Capital infraestructura }} & 0,1748 & 0,1677 & 0,1683 \\
\hline & & $(0,000)$ & $(0,000)$ & $(0,000)$ \\
\hline \multirow{2}{*}{\multicolumn{2}{|c|}{ Telecomunicaciones }} & 0,0397 & 0,0525 & 0,0460 \\
\hline & & $(0,004)$ & $(0,001)$ & $(0,001)$ \\
\hline \multirow{2}{*}{\multicolumn{2}{|c|}{ Generación eléctrica }} & 0,0962 & 0,1296 & 0,1282 \\
\hline & & $(0,134)$ & $(0,054)$ & $(0,105)$ \\
\hline \multirow{2}{*}{\multicolumn{2}{|c|}{ Líneas de tren }} & $-0,0292$ & 0,0043 & $-0,0038$ \\
\hline & & $(0,560)$ & $(0,939)$ & $(0,930)$ \\
\hline \multirow{2}{*}{\multicolumn{2}{|c|}{ Capital humano }} & 0,0015 & 0,0016 & 0,0017 \\
\hline & & $(0,000)$ & $(0,000)$ & $(0,000)$ \\
\hline \multicolumn{2}{|l|}{ Índice Starting a business } & $\begin{array}{l}0,0025 \\
(0,007)\end{array}$ & & \\
\hline \multicolumn{2}{|l|}{ Dummy índice Starting a business } & \multicolumn{2}{|r|}{$\begin{array}{l}0,0233 \\
(0,114)\end{array}$} & \\
\hline \multicolumn{2}{|l|}{$\begin{array}{l}\text { Interacción capital infraestructura } \\
\text { índice Startinga business }\end{array}$} & & & $\begin{array}{l}0,0018 \\
(0,098)\end{array}$ \\
\hline \multicolumn{2}{|l|}{$\begin{array}{l}\text { Prueba de Wald de significancia } \\
\text { conjunta (valor } \mathrm{P} \text { ) }\end{array}$} & 0 & 0 & 0 \\
\hline \multirow{2}{*}{ Test de autocorrelación (valor p) } & First order & 0,05 & 0,01 & 0,01 \\
\hline & Second order & 0,37 & 0,53 & 0,50 \\
\hline \multicolumn{2}{|l|}{$\begin{array}{l}\text { Prueba de Sargan de restricciones } \\
\text { sobre identificadas (valor } \mathrm{P} \text { ) }\end{array}$} & 1 & 1 & 1 \\
\hline \multicolumn{2}{|l|}{ No. Observaciones } & 244 & 244 & 244 \\
\hline \multicolumn{2}{|l|}{ No. Países } & 46 & 46 & 46 \\
\hline
\end{tabular}

${ }^{*}$ Valores $\mathrm{P}$ robustos (Huber y White) entre paréntesis.

Fuente: elaboración propia. 